\title{
Pulmonary involvement in acquired immunodeficiency syndrome-associated Kaposi's sarcoma: a descriptive analysis of thin-section manifestations in 29 patients
}

\author{
Chunshuang Guan ${ }^{1 \#}$, Yuxin Shi ${ }^{2 \#}$, Jinxin Liu ${ }^{3 \#}$, Yuxin Yang ${ }^{4 \#}$, Qianqian Zhang ${ }^{5 \#}$, Zhiyan Lu ${ }^{6 \#}$, \\ Guangping Zheng ${ }^{7}$, Wen $\mathrm{Ye}^{2}$, Ming Xue ${ }^{1}$, Xingang Zhou ${ }^{8}$, Na Zhang ${ }^{9}$, Hongjun Li ${ }^{10}$, Ruming Xie ${ }^{1}$, \\ Budong Chen ${ }^{1}$, Puxuan Lu ${ }^{11}$
}

${ }^{1}$ Department of Radiology, Beijing Ditan Hospital, Capital Medical University, Beijing, China; ${ }^{2}$ Department of Radiology, Shanghai Public Health Clinical Center, Shanghai, China; ${ }^{3}$ Department of Radiology, Guangzhou Eighth People's Hospital, Guangzhou Medical University, Guangzhou, China; ${ }^{4}$ Department of Radiology, The Sixth People's Hospital of Xinjiang Uygur Autonomous Region, Urumqi, China; ${ }^{5}$ Department of Radiology, Zhoukou Central Hospital, Zhoukou, China; ${ }^{6}$ Department of Radiology, Zhongnan Hospital of Wuhan University, Wuhan, China; ${ }^{7}$ Department of Radiology, The Shenzhen No. 3 People's Hospital, Guangdong Medical College, Shenzhen, China; ${ }^{8}$ Department of Pathology, Beijing Ditan Hospital, Capital Medical University, Beijing, China; ${ }^{9}$ Department of Radiology, Chengdu Public Health Clinical Center, Chengdu, China; ${ }^{10}$ Department of Radiology, Beijing You'an Hospital, Capital Medical University, Beijing, China; ${ }^{11}$ Department of Radiology, Shenzhen Center for Chronic Disease Control, Shenzhen, China

\#These authors contributed equally to this work as co-first authors.

Correspondence to: Ruming Xie. Department of Radiology, Beijing Ditan Hospital, Capital Medical University, No. 8 Jingshun East Street, Chaoyang District, Beijing, China. Email: mingrux@163.com; Budong Chen. Department of Radiology, Beijing Ditan Hospital, Capital Medical University, No. 8 Jingshun East Street, Chaoyang District, Beijing, China. Email: budongchen@sina.com; Puxuan Lu. Department of Radiology, Shenzhen Center for Chronic Disease Control, 2021\#, Buxin Road, Luohu District, Shenzhen, China. Email: 13823398132@126.com.

Background: Acquired immunodeficiency syndrome-associated Kaposi's sarcoma (AIDS-KS) was the first malignant neoplasm to be described as being related to AIDS. The lungs are the most common visceral site of AIDS-KS. This study aimed to analyze the computed tomography (CT) manifestations of pulmonary involvement in AIDS-KS.

Methods: Twenty-nine male patients were enrolled in this retrospective study. Imaging evaluation parameters included lesion distribution, the flame sign, interlobular septal thickening, peribronchovascular interstitium thickening, ground-glass opacity (GGO), dilated blood vessels in lesions, and pleural effusion.

Results: A peribronchovascular distribution was observed in all patients, predominantly in the lower lobes. Of the patients, 58.62\% (17/29) exhibited the flame sign, $75.86 \%(22 / 29)$ had interlobular septal thickening, $72.41 \%(21 / 29)$ had peribronchovascular interstitium thickening, 82.76\% (24/29) had GGO, and 34.48\% $(10 / 29)$ had pleural effusion. Enlarged lymph nodes with a short-axis diameter $>1.0 \mathrm{~cm}$ were found in $41.38 \%$ (12/29) of the patients. Of the 12 patients who underwent contrast-enhanced CT (CECT), 90.91\% (11/12) had dilated blood vessels, and nodules, consolidations, and lymph nodes were observed to be strongly enhanced. Intrapulmonary lesions decreased in size or number after appropriate treatment during follow-up. Conclusions: Common CT manifestations of pulmonary AIDS-KS include the flame sign, peribronchovascular distribution, peribronchovascular interstitium thickening, interlobular septa thickening, GGO, dilated blood vessel, and strong enhancement of nodules, consolidations, and lymph nodes. It is helpful to follow up the therapeutic effect of pulmonary AIDS-KS by chest CT.

Keywords: Acquired immunodeficiency syndrome (AIDS); lung; Kaposi's sarcoma (KS); spiral computed tomography 
Submitted Feb 15, 2020. Accepted for publication Oct 31, 2020.

doi: 10.21037/qims-20-284

View this article at: http://dx.doi.org/10.21037/qims-20-284

\section{Introduction}

Kaposi's sarcoma (KS) was first described by the Hungarian dermatologist Moritz Kaposi in 1872 (1). KS is a malignant neoplasm with vascular, lymphatic endothelial dysplasia characterized by a focal or multicenter distribution, a spindle cell composition, a split-like lumen with erythrocyte leakage, hemosiderin deposition, and dilated blood vessels (2-7). The pathology of KS can be classified into the patch, plaque, and nodular stages (2-7), and it is the first malignant neoplasm to be linked to AIDS $(8,9)$.

The incidence of KS in the general population is approximately $1 / 100,000$, compared to $1 / 20$ among individuals with human immunodeficiency virus (HIV) infection $(10,11)$. The incidence of AIDS-associated KS (AIDS-KS) increased 66-fold between 1987 and 1993 but significantly decreased after the introduction of highly active antiretroviral therapy (HAART) in 1996 (12). However, the decrease in the incidence of AIDS-KS has slowed since 2000 (13-15).

While KS can be found in the skin, oral cavity, digestive tract, lymph nodes, bone, lung, and liver $(5,16)$, pulmonary involvement (pulmonary AIDS-KS) is the most common visceral manifestation and can be found in $47 \%$ of AIDSKS patients at autopsy (17). Chest computed tomography (CT) is the most commonly used method in the diagnosis of pulmonary AIDS-KS. In our previous work, we observed localized dilated blood vessels as a common manifestation of pulmonary AIDS-KS on contrast-enhanced CT (CECT), which is a finding not mentioned by other studies $(11,13,18,19)$. This retrospective study aimed to analyze the CT manifestations of pulmonary involvement in patients with AIDS-KS.

\section{Methods}

\section{Study subjects}

This study was approved by the Institutional Ethics Review Board of Beijing Ditan Hospital, Capital Medical University. The requirement for informed consent was waived due to the analysis being retrospective. A flow chart of enrolled patients is shown in Figure 1. Data on 85 patients suspected of AIDS-KS were collected from six infectious disease hospitals between January 1, 2016 and January 28, 2020. The inclusion criteria were: (I) positive HIV antibody result of initial screening and confirmatory testing; (II) clinical presentation suggested AIDS-KS; (III) CT showed abnormal chest manifestations and AIDS-KS could not be excluded; (IV) KS was confirmed by pathology; and $(\mathrm{V})$ follow-up showed improvement or progression after treatment. The exclusion criteria were: (I) KS could not be confirmed by pathology (4/85, 4.71\%); (II) normal chest CT manifestations (12/85, 14.12\%); (III) pulmonary infections (33/85, 38.82\%); and (IV) assessment affected by imaging quality $(7 / 85,8.24 \%)$ (Figure 1).

Finally, 29 male patients $(29 / 85,34.12 \%)$ were enrolled in the study. The patients were aged between 19 and 52 years, and had a mean age of $36.21 \pm 8.78$ years. The median duration of HIV antibody positive status was 5 [2, 24] months. Twenty-one patients (21/29, 72.41\%) presented with respiratory symptoms, including cough, expectoration, chest distress, and dyspnea, while 8 patients $(8 / 29,27.59 \%)$ had no respiratory symptoms. All patients showed skin or oral nodules which were progressively enlarging. Nodules and masses were present for a median duration of 4 [2, 11] months. The nodules and masses gradually deepened in color, from red to purple, and lymph nodes of the neck or inguinal regions increased in size in four patients. Aside from pulmonary involvement, all 29 patients $(100.0 \%$, 29/29) had skin involvement. Also, the liver was involved in 7 patients $(24.14 \%, 7 / 29)$ (Figure 2), the oral cavity in 4 patients $(13.79 \%, 4 / 29)$, the lymph nodes in 4 patients $(13.79 \%, 4 / 29)$, and the spine, ribs, humerus, and scapula in 1 patient $(3.45 \%, 1 / 29)$.

Among the 12 patients (41.38\%) who were followed up, 8 were treated simultaneously with chemotherapy and HAART $(66.67 \%, 8 / 12), 3$ received HAART only $(25.00 \%$, $3 / 12)$, and 1 received chemotherapy only $(8.33 \%, 1 / 12)$.

\section{Pathology}

AIDS-KS was confirmed by lung needle biopsy and skin needle biopsy in 8 cases $(27.59 \%, 8 / 29)$, by bronchoscopy biopsy and skin needle biopsy in 4 cases $(13.79 \%, 4 / 29)$, by lymph node needle biopsy in 3 cases $(10.34 \%, 3 / 29)$, by skin needle biopsy alone in 12 cases $(41.38 \%, 12 / 29)$, and by oral 


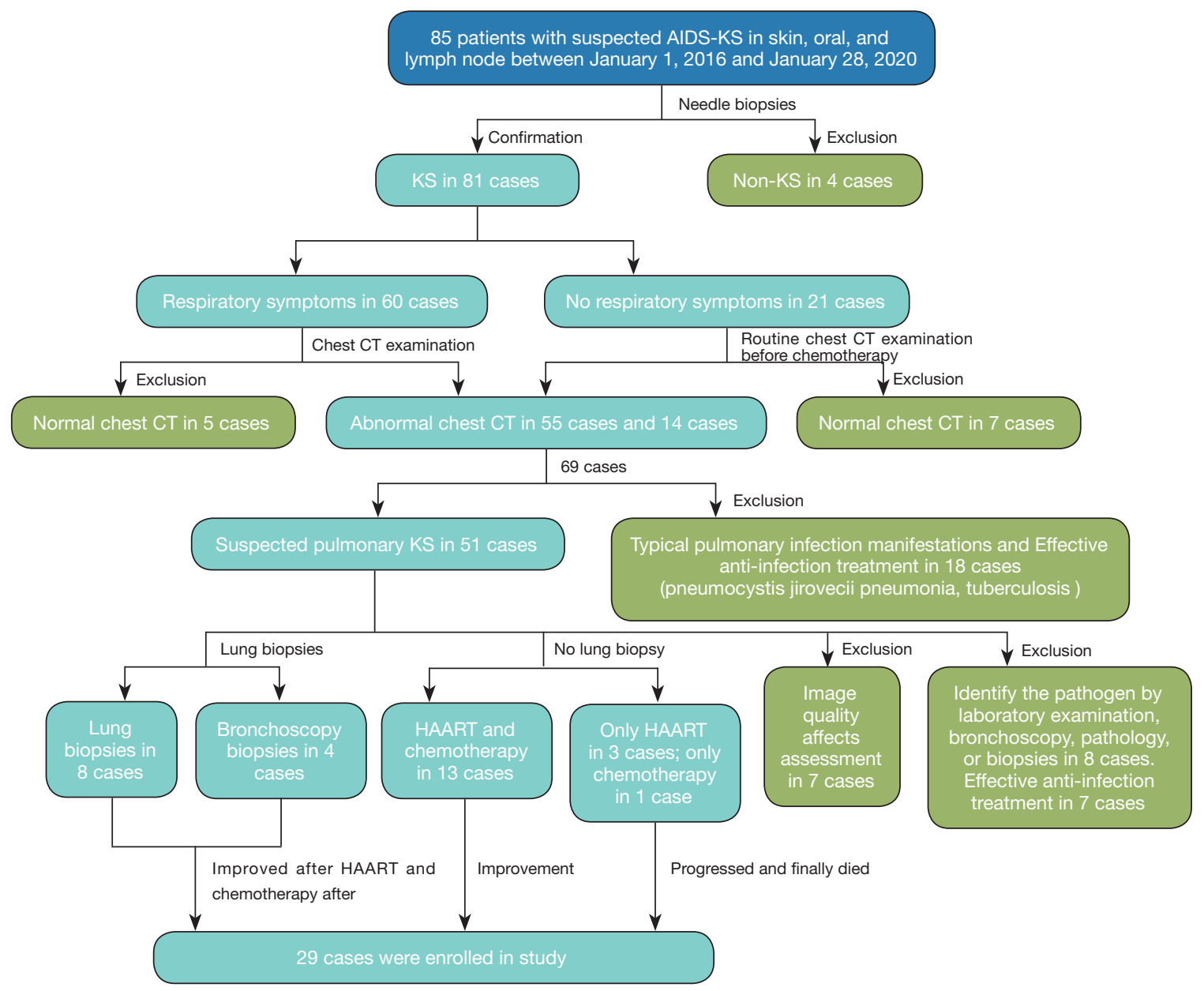

Figure 1 Flowchart of enrolled patients. KS, Kaposi's sarcoma; AIDS-KS, acquired immunodeficiency syndrome-associated Kaposi's sarcoma; CT, computed tomography; HAART, highly active antiretroviral therapy.

needle biopsy in 2 cases $(6.90 \%, 2 / 29)$.

The biopsy tissues were fixed in formalin, embedded in paraffin, and cut into $5-\mu \mathrm{m}$ sections. The sections were then stained with hematoxylin and eosin (HE) and observed under a microscope. Immunohistochemical staining with EnVision was then performed for CD34 cytoplasm and human herpesvirus (HHV)-8 cell nuclei in tumor cells. The positive expression of CD34 suggested that the tumor was of vascular origin and the positive expression of HHV-8 suggested that the tumor was KS $(3-5,9,19)$. On the basis that the pathology of $\mathrm{KS}$ is classified into patch, plaque, and nodular stages (2-7), all patients in this study had reached the nodular stage. On microscopy, a large number of spindle cells were observed as nodular hyperplasia partially arranged into a split-like lumen, from which erythrocytes leaked out. Scattered lymphocytes and plasma cell infiltration were observed in the interstitium. Immunohistochemistry showed staining of the CD34 cytoplasm of some spindle cells and HHV-8 cell nuclei of spindle cells.

\section{CT scanning equipment and methods}

All chest CT scans were performed on patients in the supine position at the end of inspiration, within 2 weeks of biopsy and before chemotherapy (Figure 1). No cases of infection or other new symptoms were reported within the 2 weeks. Scanning equipment included 16-slice, 64-slice, and 128-slice spiral CTs (Siemens, sensation CT, Forchheim, Germany; GE, Light speed vCT, Wisconsin, USA; Philips, iCT, The Netherlands). The scanning ranged from apex to base, and the scanning parameters included tube voltage of $120 \mathrm{kV}$, automatic tube current, and a slice thickness of $1.25 \mathrm{~mm}$. 

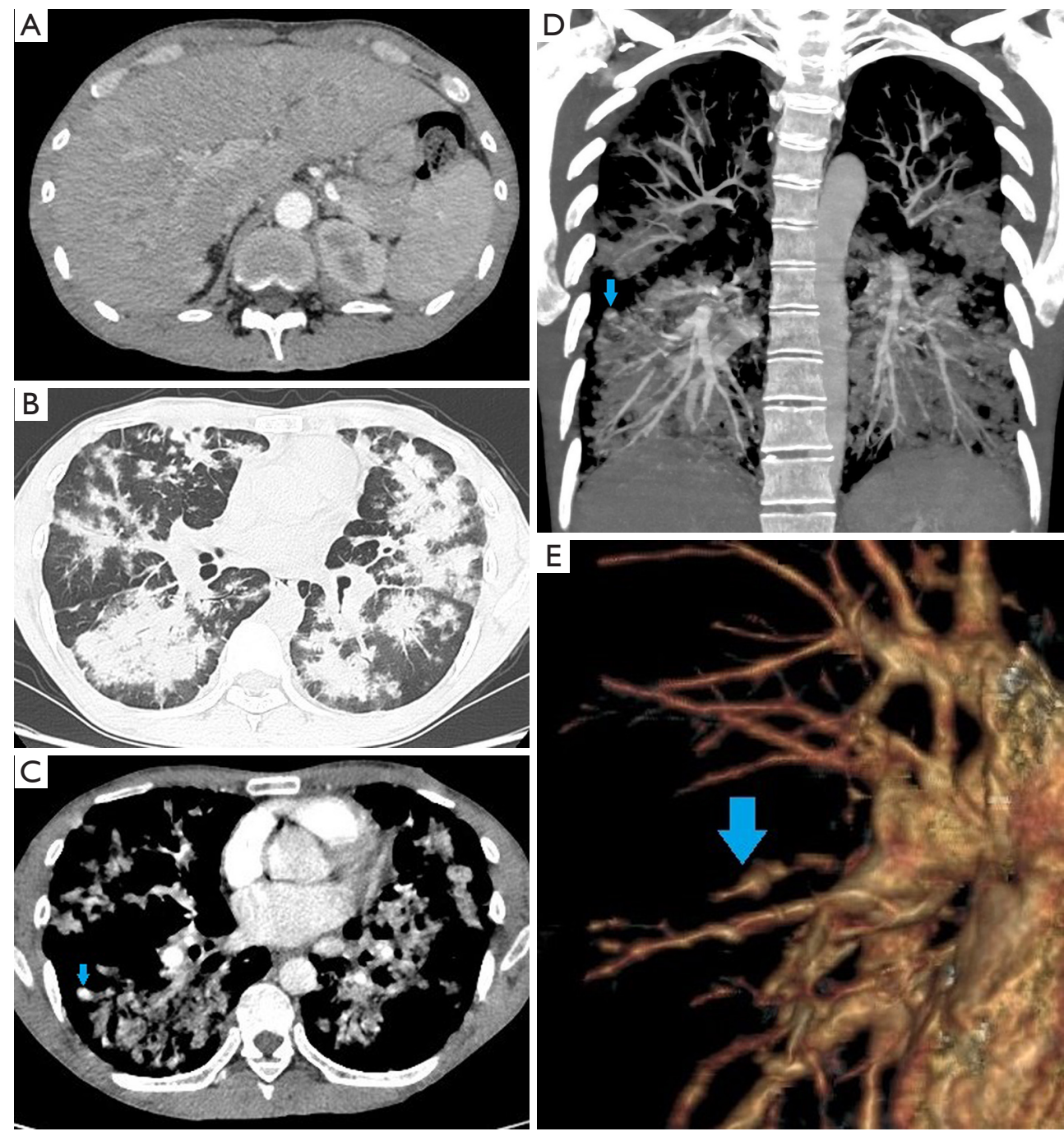

Figure 2 Pulmonary involvement in a 41-year-old male with acquired immunodeficiency syndrome-associated Kaposi's sarcoma. (A) The patient's liver involvement is shown as an annular enhanced nodular lesion along the portal vein on the contrast-enhanced CT image; (B) a CT scan of the lung window shows flame signs and peribronchovascular interstitial thickening with symmetrical distribution. Ground-glass opacity and interlobular septal thickening can be observed around the lesions; $(\mathrm{C})$ the lesions located around the vessels are shown to be strongly enhanced on the contrast-enhanced CT scan, with dilated blood vessels (arrow) in the lesions; (D) a maximum intensity projection showing lesions distributed along the peribronchovascular bundle, and dilated blood vessels (arrow); (E) volume rendering showing uneven lumens of the pulmonary artery branches and local dilated blood vessels (arrow). CT, computed tomography.

\section{Imaging assessment}

The whole lung CT images were evaluated by two chest radiologists (with professional experience of 11 and 12 years, respectively) on the picture archiving and communication system workstation, and any disagreements were settled through discussions. Imaging manifestation parameters included: (I) peribronchovascular distribution; (II) lung lobe involvement; (III) the flame sign (presented as a nodule or consolidation with ill-defined margins; Figure 2); (IV) morphology (nodules or patchy consolidation); (V) peribronchovascular interstitium thickening; (VI) interlobular septal thickening; (VII) ground-glass opacity (GGO); (VIII) air bronchogram; (IX) the short-axis diameter of the largest lymph node in the mediastinum; (X) pleural effusion and pericardial effusion; (XI) dilated blood vessels in nodules or consolidations on CECT; (XII) the 
densities of nodules or consolidations and lymph nodes on plain CT and CECT; (XIII) measurements were obtained by the two radiologists, and the mean values were used as the final measurement values. The short-axis diameters and densities of the largest lymph node in the mediastinum were measured on plain CT and CECT. The densities of intrapulmonary lesions (the largest nodule or consolidation) were measured at the same position on plain CT and CECT, and the vessels on CECT were avoided.

\section{Statistical analysis}

Statistical analyses were performed using SPSS 17.0 software. Continuous data were expressed as the mean and standard deviation or median (25th percentile, 75 th percentile), and categorical data were expressed as the frequency. The Mann-Whitney $U$ test was used to analyze statistical differences in the density of intrapulmonary lesions and lymph nodes between plain CT and CECT. A P value of $<0.05$ was considered to be statistically significant.

\section{Results}

\section{Distribution}

All 29 patients showed peribronchovascular distribution involving all pulmonary lobes. Twenty-three patients exhibited more lesions in the lower lobes $(79.31 \%)$ while 6 (20.69\%) showed no distribution tendency (Figures 2-4).

\section{Imaging manifestations on plain $C T$}

The flame sign was found in 17 patients $(58.62 \%), 22$ patients showed interlobular septal thickening $(75.86 \%)$, 21 showed peribronchovascular interstitium thickening (72.41\%), 24 showed GGO (82.76\%), 15 showed nodules (51.72\%), 14 showed consolidation (48.28\%), and 21 showed air bronchogram (72.41\%). All GGOs were located around nodules or consolidations. Small-to-moderate amounts of pleural effusion $(34.48 \%, 10 / 29)$ and small amounts of pericardial effusion $(10.34 \%, 3 / 29)$ were found. Ten patients had large patchy consolidation (34.48\%) and 1 presented large patchy GGO (3.45\%) (Table 1 and Figures 2-4)

The largest lymph node involved the 4R (48.28\%, 14/29), $5(24.14 \%, 7 / 29), 7(27.59 \%, 8 / 29)$, and $10 \mathrm{~L}(3.45 \%$, $1 / 29)$ regions in the mediastinum. The mean short-axis diameter of the largest lymph nodes was $1.005 \pm 0.176 \mathrm{~cm}$ (range, 0.78-1.29 cm), and 12 patients $(41.38 \%, 12 / 29)$ had a short-axis diameter $>1.0 \mathrm{~cm}$.

\section{Imaging manifestations on CECT}

CECT was performed on 12 patients. Dilated blood vessels in nodules or consolidations were observed in 11 of them (90.91\%) (Figures 2,3), with only one patient presenting with small well-defined nodules without dilated blood vessels being found (Figure 4). Intrapulmonary lesions (nodules or consolidations) and lymph nodes were observed to be strongly enhanced, with an enhancement degree of $41.103 \pm 17.169 \mathrm{HU}$ and $49.177 \pm 15.133 \mathrm{HU}$, respectively (Table 2). The degree of enhancement did not significantly differ between intrapulmonary lesions and lymph nodes $(\mathrm{P}=0.340)$, nor was any significant difference found in density between the intrapulmonary lesions and lymph nodes, regardless of whether plain CT or CECT was used $(\mathrm{P}=0.387$ and $\mathrm{P}=0.222$, respectively) (Table 2).

\section{Follow-up}

Twelve patients were followed up, and the mean time interval was $3.08 \pm 3.42$ months $(0.50-12.00$ months). Eight patients $(66.67 \%)$, who were treated with $6.25 \pm 4.23$ courses (0.30-11.37 courses) of chemotherapy and continuous HAART, showed a decrease in size or number of intrapulmonary lesions (including nodules, consolidation, peribronchovascular interstitium thickening, interlobular septal thickening, and GGO), including one patient who exhibited complete resolution (8.33\%) (Figure 3). The other four patients, who were treated with continuous HAART only or 1 course of chemotherapy only, showed an increase in size and number of intrapulmonary lesions $(33.33 \%, 4 / 12)$ and eventually died. One of these patients $(25.00 \%, 1 / 4)$ showed nodule progression to consolidation and the other 3 showed an increase in size and number of nodules and consolidations. Pleural effusion was completely absorbed in 3 patients $(25.00 \%)$, decreased in 2 patients $(16.67 \%)$, and increased in 3 patients $(25.00 \%)$, while no change was observed in 4 patients $(33.33 \%)$. No changes were observed in pericardial effusion. Lymph nodes were enlarged in 2 patients $(16.67 \%)$, reduced in $1(8.33 \%)$, and remained unchanged in 9 patients $(75.00 \%)$.

Only 1 of the 12 follow-up patients underwent CECT $(8.33 \%, 1 / 12)$, the other patients underwent plain CT. This patient was followed up after 8 months of treatment with 12 courses of chemotherapy and continuous HAART 

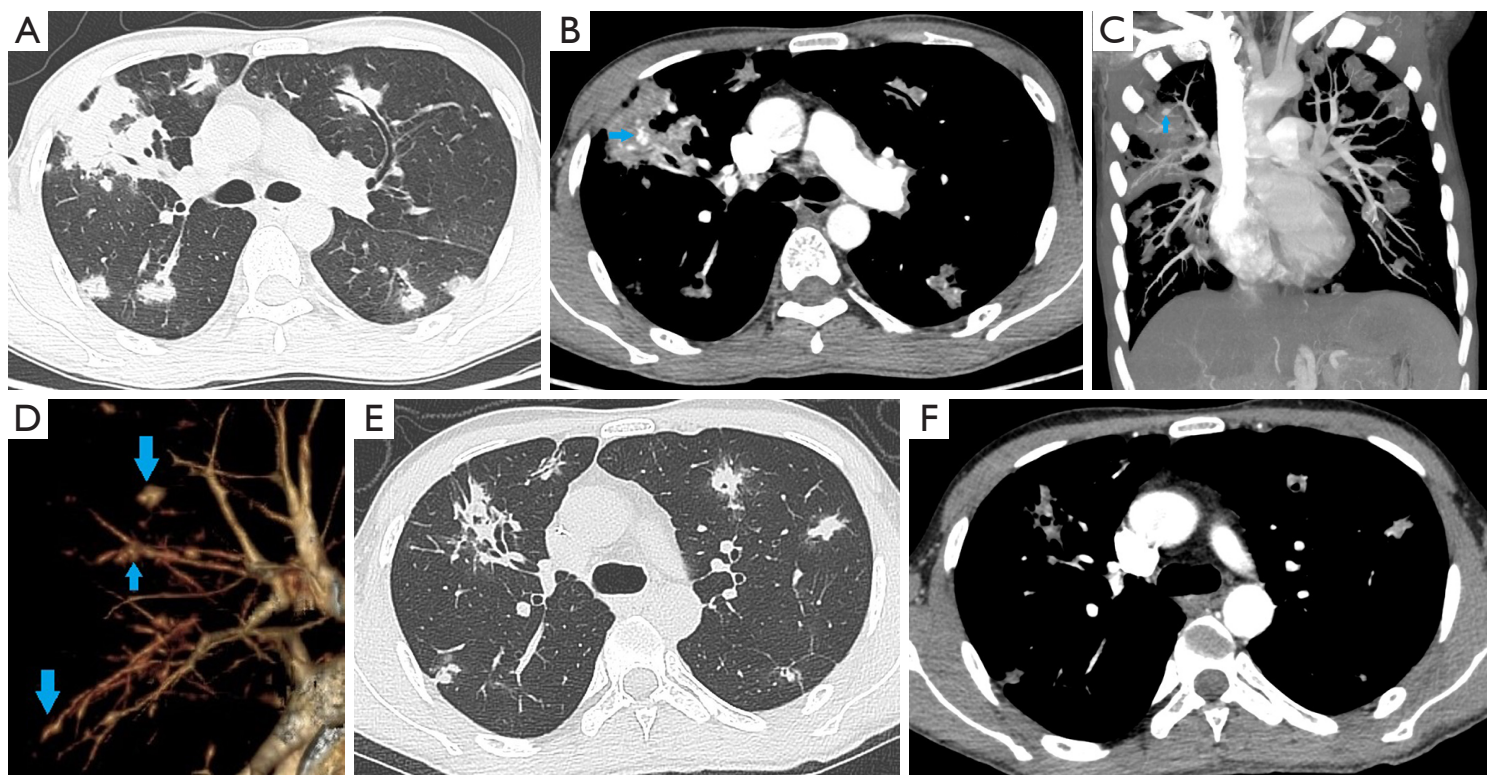

G
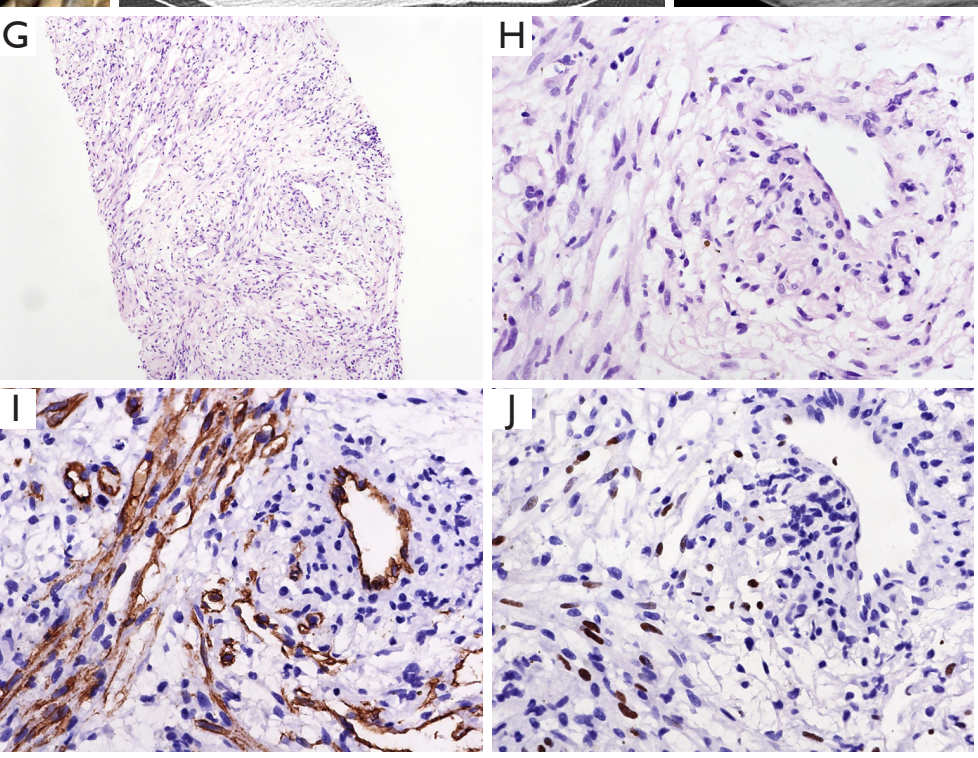

Figure 3 A 33-year-old male with pulmonary involvement in acquired immunodeficiency syndrome-associated Kaposi's sarcoma. CT imaging manifestations, histopathology, and immunohistochemistry findings of the lung biopsy are shown. (A) A CT scan of the lung window showing flame signs distributed asymmetrically along the peribronchovascular bundle. Ground-glass opacity can be observed at the margin of nodules and consolidation. (B) A contrast-enhanced CT scan showing strong enhancement of nodules and consolidation. Dilated blood vessels (arrow) can be observed in the consolidation in the anterior segment of the upper lobe in the right lung. (C) A maximum intensity projection showing lesions distributed along the peribronchovascular bundle, with dilated blood vessels (arrow) in the lesions. (D) Volume rendering showing dilated blood vessels (arrow) at the end of the arterial vessels. (E) Intrapulmonary lesions significantly decreased after treatment for 1.5 months. Flame signs can be seen. (F) A contrast-enhanced CT scan performed after 1.5 months of treatment shows that the previous dilated lumen in the lesion returned to normal (as compared with Figure 1B). (G) Damage to the normal lung structure shown in HE $\times 100$ biopsy tissue, and a large number of atypical spindle cells are shown as nodular hyperplasia, partially arranged into a split-like lumen. (H) In HE $\times 400$ biopsy tissue, a large number of atypical spindle cells are shown as nodular hyperplasia, partially arranged into a split-like lumen; scattered effusion of erythrocytes; scattered lymphocytes and plasma cell infiltration can be seen in the interstitium. (I) The image shows that the CD34 cytoplasm of some spindle cells was stained after immunohistochemical staining with EnVision $\times 400$. (J) An image showing stained HHV-8 cell nuclei after immunohistochemical staining with EnVision $\times 400$. CT, computed tomography. 

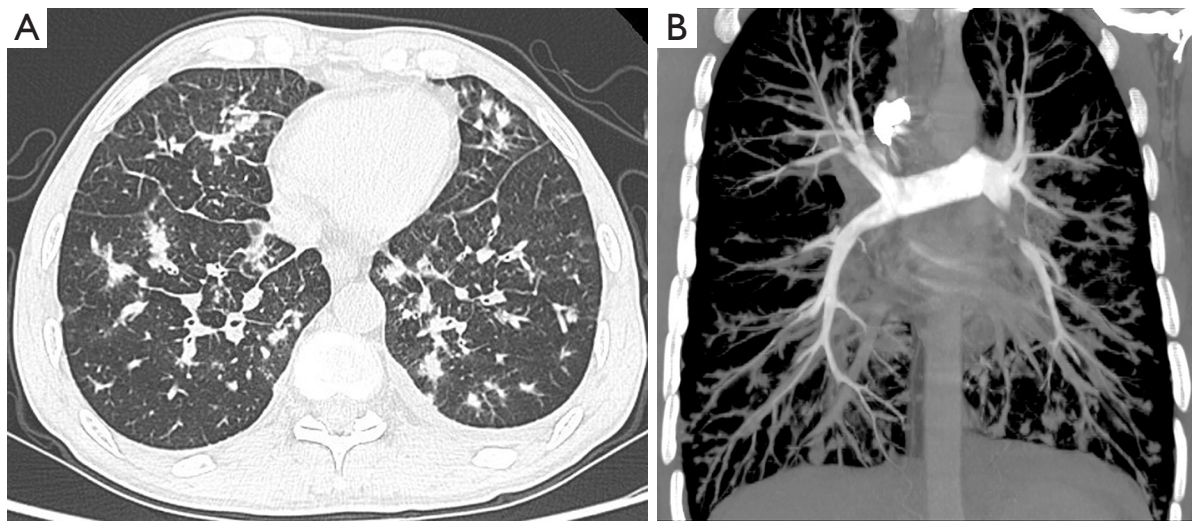

Figure 4 A 47-year-old with pulmonary involvement in acquired immunodeficiency syndrome-associated Kaposi's sarcoma. (A) A CT scan of the lung window showing flame signs symmetrically distributed along the peribronchovascular bundle in both lungs. Interlobular septal thickening can be seen around nodules. (B) A maximum intensity projection showing nodules distributed along the blood vessels, with uniform lumens of vessels and no shadows of dilated vessels. CT, computed tomography.

Table 1 Prevalence of imaging findings of 29 patients with pulmonary involvement in acquired immunodeficiency syndrome-associated Kaposi's sarcoma

\begin{tabular}{lc}
\hline Image signs & Number of patients (\%) \\
\hline Nodule & $15(51.72)$ \\
Consolidation & $14(48.28)$ \\
All lobes involved & $29(100.0)$ \\
Distribution of bronchial vascular bundle & $29(100.0)$ \\
Flame sign & $17(58.62)$ \\
Bronchovascular interstitium thickening & $21(72.41)$ \\
Interlobular septal thickening & $22(75.86)$ \\
Ground-glass opacity & $24(82.76)$ \\
Air bronchogram & $21(72.41)$ \\
Pleural effusion & $10(34.48)$ \\
Pericardial effusion & $3(10.34)$ \\
\hline
\end{tabular}

treatment at which point their dilated vessels returned to the normal range (Figure 3).

\section{Discussion}

Pulmonary KS can involve the pulmonary parenchyma, tracheobronchial tree, pleura, and mediastinal lymph nodes $(20,21)$. Previous studies have shown that in its early stage, the disease causes peribronchovascular interstitium thickening, especially at the base of the lungs
Table 2 The density of intrapulmonary lesions and lymph nodes before and after contrast-enhanced computed tomography for pulmonary involvement in acquired immunodeficiency syndrome-associated Kaposi's sarcoma

\begin{tabular}{lccc}
\hline Variable & Plain CT $(\mathrm{HU})$ & $\begin{array}{c}\text { Contrast- } \\
\text { enhanced CT } \\
(\mathrm{HU})\end{array}$ & $\begin{array}{c}\text { Increased CT } \\
\text { density after } \\
\text { enhanced }(\mathrm{HU})\end{array}$ \\
\hline $\begin{array}{l}\text { Intrapulmonary } \\
\text { lesions }\end{array}$ & $30.073 \pm 7.404$ & $71.203 \pm 16.930$ & $41.103 \pm 17.169$ \\
Lymph node & $34.241 \pm 6.878$ & $83.418 \pm 17.883$ & $49.177 \pm 15.133$ \\
P value $^{*}$ & 0.387 & 0.222 & 0.340 \\
\hline
\end{tabular}

*, $\mathrm{P}$ value refers to intrapulmonary lesions vs. lymph node. HU, Hounsfield unit.

$(22,23)$, and this was also observed frequently in the present study $(72.41 \%)$. The chest CT manifestations of KS also change as the disease progresses $(22,23)$. The flame sign is commonly seen as it distributes along the peribronchovascular bundle $(13,18)$, and it was observed in $58.62 \%$ of patients in the present study. GGO can surround nodules and consolidations $(24,25)$, and this was also seen in almost half of patients in the present study, with $34.48 \%$ of patients showing large patchy consolidation. Previous studies have also showed that nodules can progress to consolidation (24), which was observed in 1 of the 12 patients who were followed up in the present study. Interlobular septal thickening, which commonly appears in KS patients $(22,23)$, was found in $75.86 \%$ of patients in this study. Enlarged lymph nodes are seen in $30 \%$ to $35 \%$ of KS 
patients $(11,19)$, which is close to the incidence reported in the present study (41.38\%). Pleural effusion is also a common manifestation of KS $(22,23)$, occurring in $66.7 \%$ of KS patients with lung parenchyma involvement (26), and was found in $34.48 \%$ of patients in this study. Moderateto-large pericardial effusions occasionally occur in KS patients (27-30). Small amounts of pericardial effusion are commonly seen in the normal population. In the present study, this was seen in 3 patients (10.34\%) and remained unchanged after treatment, indicating that this may have been unrelated to AIDS-KS. Biopsy would be necessary to make to make a definitive pathologic diagnosis (31).

Dilated blood vessels in consolidation and nodules were commonly observed on CECT (90.91\%) in our study, although this has not been described in previous studies $(11,13,18,19)$. Some studies of pulmonary AIDS-KS have referred to significant intrapulmonary enhancement in figures but have not described the enhanced manifestation (23,32-34). One study of the magnetic resonance imaging (MRI) manifestation of pulmonary AIDS-KS described strong enhancement on contrast-enhanced MRI (35). Some visceral KSs present as an enhanced mass, which is commonly associated with enhanced regional lymph nodes (23). Our observation of dilated blood vessels, which was not described in previous studies $(11,13,18,19)$, might be explained by the increased scanning speed, increased density resolution, and thinner scanning layer of current CT. On this basis, we performed CECT scans before lung biopsies to avoid vessel puncture and the risk of hemorrhage. Only in 1 patient, who received followup CECT after 8 months of treatment, were the dilated vessels observed to have returned to their normal diameters. Therefore, further research is required to investigate this observation. The enhancement degree of lymph nodes was higher than that of nodules and consolidations; however, a statistically significant difference was not found, which may be attributable to the small sample size.

A better understanding of the pathological basis of KS would facilitate a better understanding of its imaging manifestations. The neovascularization seen in KS before the formation of a mass is different from that in other malignant neoplasms $(10,19)$. The interstitial thickening of the bronchovascular bundle and interlobular septal thickening are caused by lymphatic obstruction or tumor invasion $(13,18)$. The flame sign corresponds to the pathologically nodular hyperplasia of spindle cells, lymphedema, and peripheral inflammatory cells $(4,5,7)$. GGO is caused by hemorrhage or exudation in an adjacent area (36), while enlarged lymph nodes might be associated with lymphatic involvement $(11,19)$. Pleural effusion may be unilateral or bilateral, exudate or leakage, and is usually bloody in appearance (28). Likewise, pericardial effusion is commonly exudative and bloody (27-30). Dilated blood vessels can be observed in all pathologic stages of KS (3-7), which was in line with the common CT manifestation of local dilated blood vessels on CECT in this study. The obvious enhancement of nodules or consolidations on CECT may be explained by the inflammation, vascular proliferation, blood vessel dilation, and split-like lumen (3-7).

Unlike other imaging findings such as nodules, consolidation, thickened peribronchovascular interstitium, thickened interlobular septa, GGO, dilated blood vessels, and strong enhancement, the flame sign is a characteristic sign of AIDS-KS. However, studies have reported that the combination of the imaging manifestations of pulmonary AIDS-KS is characteristic $(25,37)$, which can help to make an accurate diagnosis.

In the follow-up, the nodules, consolidation, thickened peribronchovascular interstitium, thickened interlobular septa, GGO, pleural effusion, and lymph nodes decreased or increased in size or number after different treatment schedules. Therefore, it was convenient to observe the therapeutic response and effect using chest CT, which could help clinicians to provide appropriate treatment for their patients.

It is important to differentiate pulmonary AIDS-KS from lymphoma and infectious diseases. Lymphoma, which is the most common tumor in patients with AIDS, shows consolidation, nodules, masses, and air bronchogram. Lymphoma also shows mild-to-moderate enhancement on CECT and angiographic signs $(31,38,39)$. However, the peribronchovascular distribution, flame sign, and interlobular septal thickening present in AIDSKS differentiate it from lymphoma (40). The dilated blood vessels and strong enhancement observed in this study might also be helpful in differential diagnosis. Galliumthallium radionuclide imaging can be used to distinguish KS from infectious or lymphoma in AIDS patients. KS is thallium avid and does not take up gallium, while infectious diseases and lymphoma take up gallium but not thallium (41).

There are several limitations to this study. First, the sample size was small and sampling error was unavoidable, which might be due to the low proportion of lung involvement in AIDS-KS. Further, the study could not 
confirm the pathological basis of every CT manifestation in pulmonary AIDS-KS. Further study is needed to address these issues.

In conclusion, the flame sign with peribronchovascular distribution, peribronchovascular interstitium thickening, interlobular septal thickening, GGO, dilated blood vessels, and strong enhancement were common CT manifestations in patients with AIDS-KS. CECT is required not only for making a diagnosis but also for avoiding hemorrhage caused by punctures. The use of follow-up chest CT could help clinicians to adopt the most appropriate therapeutic schedule for patients.

\section{Acknowledgments}

We thank the Beijing Deep Intelligent Pharma Technologies Co., Ltd for providing language editing services.

Funding: None.

\section{Footnote}

Conflicts of Interest: All authors have completed the ICMJE uniform disclosure form (available at http://dx.doi. org/10.21037/qims-20-284). The authors have no conflicts of interest to declare.

Ethical Statement: This study was approved by the Institutional Ethics Review Board (Beijing Ditan Hospital, Capital Medical University). Informed consent was waived for this retrospective analysis.

Open Access Statement: This is an Open Access article distributed in accordance with the Creative Commons Attribution-NonCommercial-NoDerivs 4.0 International License (CC BY-NC-ND 4.0), which permits the noncommercial replication and distribution of the article with the strict proviso that no changes or edits are made and the original work is properly cited (including links to both the formal publication through the relevant DOI and the license). See: https://creativecommons.org/licenses/by-nc-nd/4.0/.

\section{References}

1. Kaposi M. Idiopathisces multiples Pigmentsarkom der Haut. Archiv für Dermatologie und Syphilis 1872;3:265-73.

2. Katabathina VS, Menias CO, Tammisetti VS, Lubner MG, Kielar A, Shaaban A, Mansour J, Surabhi VR, Hara
AK. Malignancy after Solid Organ Transplantation: Comprehensive Imaging Review. Radiographics 2016;36:1390-407.

3. Kaplan LD. Human herpesvirus-8: Kaposi sarcoma, multicentric Castleman disease, and primary effusion lymphoma. Hematology Am Soc Hematol Educ Program 2013;2013:103-8.

4. Flore O, Rafii S, Ely S, O’Leary JJ, Hyjek EM, Cesarman E. Transformation of primary human endothelial cells by Kaposi's sarcoma-associated herpesvirus. Nature 1998;394:588-92.

5. Katano H. Pathological Features of Kaposi's SarcomaAssociated Herpesvirus Infection. Adv Exp Med Biol 2018;1045:357-76.

6. Mouden K, Khmou M, Loughmari S, Semmar A, El Kacemi H, El Khannoussi B, Kebdani T, Elmajjaoui S, Benjaafar N. Primary Kaposi's sarcoma of the nasal cavity: a case report and review of the literature. Clin Sarcoma Res 2016;6:4.

7. Valentin RG, Drew PA, Benninger LA, Tunsupon P. Endobronchial Kaposi Sarcoma. J Bronchology Interv Pulmonol 2019;26:62-5.

8. Hernández-Ramírez RU, Shiels MS, Dubrow R, Engels EA. Cancer risk in HIV-infected people in the USA from 1996 to 2012: a population-based, registry-linkage study. Lancet HIV 2017;4:e495-504.

9. Mariggiò G, Koch S, Schulz TF. Kaposi sarcoma herpesvirus pathogenesis. Philos Trans R Soc Lond B Biol Sci 2017;372:20160275.

10. La Ferla L, Pinzone MR, Nunnari G, Martellotta F, Lleshi A, Tirelli U, De Paoli P, Berretta M, Cacopardo B. Kaposi's sarcoma in HIV-positive patients: the state of art in the HAART-era. Eur Rev Med Pharmacol Sci 2013;17:2354-65.

11. Hashmi HR, Niazi M, Adrish M. A Woman in Her 30s With a Past History of HIV Disease Presented With Recurrent Fever, Night Sweats, and Small Bilateral Pulmonary Nodules. Chest 2016;149:e177-82.

12. Hiatt KM, Nelson AM, Lichy JH, Fanburg-Smith JC. Classic Kaposi sarcoma in the United States over the last two decades: a clinicopathologic and molecular study of 438 non-HIV-related Kaposi sarcoma patients with comparison to HIV-related Kaposi sarcoma. Mod Pathol 2008;21:572-82.

13. Marcoval J, Bonfill-Ortí M, Martínez-Molina L, ValentíMedina F, Penín RM, Servitje O. Evolution of Kaposi sarcoma in the past 30 years in a tertiary hospital of the European Mediterranean basin. Clin Exp Dermatol 
2019;44:32-9.

14. Shiels MS, Islam JY, Rosenberg PS, Hall HI, Jacobson E, Engels EA. Projected cancer incidence rates and burden of incident cancer cases in HIV-infected adults in the United States through 2030. Ann Intern Med 2018;168:866-73.

15. Park LS, Tate JP, Sigel K, Rimland D, Crothers K, Gibert C, Rodriguez-Barradas MC, Goetz MB, Bedimo RJ, Brown ST, Justice AC, Dubrow R. Time trends in cancer incidence in persons living with HIV/AIDS in the antiretroviral therapy era: 1997-2012. AIDS 2016;30:1795-806.

16. Ozdemir E, Poyraz NY, Keskin M, Kandemir Z, Turkolmez S. (18)F-FDG PET/CT findings in a case with HIV (-) Kaposi sarcoma. Rev Esp Med Nucl Imagen Mol 2014;33:175-7.

17. Meduri GU, Stover DE, Lee M, Myskowski PL, Caravelli JF, Zaman MB. Pulmonary Kaposi's sarcoma in the acquired immune deficiency syndrome. Clinical, radiographic, and pathologic manifestations. Am J Med 1986;81:11-8.

18. Gasparetto TD, Marchiori E, Lourenço S, Zanetti G, Vianna AD, Santos AA, Nobre LF. Pulmonary involvement in Kaposi sarcoma: correlation between imaging and pathology. Orphanet J Rare Dis 2009;4:18.

19. Ganem D. KSHV and the pathogenesis of Kaposi sarcoma: listening to human biology and medicine. J Clin Invest 2010;120:939-49.

20. Lodi S, Guiguet M, Costagliola D, Fisher M, de Luca A, Porter K; CASCADE Collaboration. Kaposi sarcoma incidence and survival among $\mathrm{HIV}$-infected homosexual men after HIV seroconversion. J Natl Cancer Inst 2010;102:784-92.

21. Alwassia A, Alshathri Z, Khosla R, Spagnolo SV. Pulmonary Kaposi sarcoma presenting as complete lung consolidation. BMJ Case Rep 2017;2017:bcr2016219048.

22. Aboulafia DM. The epidemiologic, pathologic, and clinical features of AIDS-associated pulmonary Kaposi's sarcoma. Chest 2000;117:1128-45.

23. Restrepo CS, Ocazionez D. Kaposi's sarcoma: imaging overview. Semin Ultrasound CT MR 2011;32:456-69.

24. Javadi S, Menias CO, Karbasian N, Shaaban A, Shah K, Osman A, Jensen CT, Lubner MG, Gaballah AH, Elsayes KM. HIV-related Malignancies and Mimics: Imaging Findings and Management. Radiographics 2018;38:2051-68.

25. Traill ZC, Miller RF, Shaw PJ. CT appearances of intrathoracic Kaposi's sarcoma in patients with AIDS. Br J
Radiol 1996;69:1104-7.

26. Allen CM, Al-Jahdali HH, Irion $\mathrm{KL}, \mathrm{Al}$ Ghanem S, Gouda A, Khan AN. Imaging lung manifestations of HIV/AIDS. Ann Thorac Med 2010;5:201-16.

27. Stotka JL, Good CB, Downer WR, Kapoor WN. Pericardial effusion and tamponade due to Kaposi's sarcoma in acquired immunodeficiency syndrome. Chest 1989;95:1359-61.

28. Gouny P, Lancelin C, Girard PM, Hocquet-Cheynel C, Rozenbaum W, Nussaume O. Pericardial effusion and AIDS: benefits of surgical drainage. Eur J Cardiothorac Surg 1998;13:165-9.

29. Chen Y, Brennessel D, Walters J, Johnson M, Rosner F, Raza M. Human immunodeficiency virus-associated pericardial effusion: report of 40 cases and review of the literature. Am Heart J 1999;137:516-21.

30. Lababidi MH, Alhawasli H, Iroegbu N. Kaposi sarcoma can also involve the heart. J Community Hosp Intern Med Perspect 2015;5:29054.

31. Chen L, Liu M, He XQ, Wu YS, Li QS, Chen YK. Acquired immunodeficiency syndrome (AIDS) related lymphoma: 29 clinical cases and the factors influencing their prognosis. Electronic Journal of Emerging Infectious Diseases 2018;3:153-5.

32. Habib SA, Vasko RC, Badawy J, Anstead GM. Plastic Bronchitis in an AIDS Patient with Pulmonary Kaposi Sarcoma. Case Rep Pulmonol 2018;2018:9736516.

33. Dirweesh A, Khan MY, Hamiz SF, Karabulut N. Pulmonary Kaposi Sarcoma with Osseous Metastases in a Human Immunodeficiency Virus (HIV) Patient: A Remarkable Response to Highly Active Antiretroviral Therapy. Am J Case Rep 2017;18:181-5.

34. Petribu NC, Cisneiros MS, Carvalho GB, et al. Pulmonary Kaposi's sarcoma in a female patient: Case report. Rev Assoc Med Bras (1992) 2016;62:395-8.

35. Khalil AM, Carette MF, Cadranel JL, Mayaud CM, Akoun GM, Bigot JM. Magnetic resonance imaging findings in pulmonary Kaposi's sarcoma: a series of 10 cases. Eur Respir J 1994;7:1285-9.

36. da Silva Filho FP, Marchiori E, Valiante PM, Escuissato DL, Gasparetto TD. AIDS-related Kaposi sarcoma of the lung presenting with a "crazy-paving" pattern on highresolution CT: imaging and pathologic findings. J Thorac Imaging 2008;23:135-7.

37. Khalil AM, Carette MF, Cadranel JL, Mayaud CM, Bigot JM. Intrathoracic Kaposi's sarcoma. CT findings. Chest 1995;108:1622-6.

38. Bae YA, Lee KS, Han J, Ko YH, Kim BT, Chung MJ, 
Kim TS. Marginal zone B -cell lymphoma of bronchusassociated lymphoid tissue: imaging findings in 21 patients. Chest 2008;133:433-40.

39. Luo Y, Lu P, Le X, Luo X. Radiological and histological findings characteristic of AIDS related Burkitt lymphoma. Radiol Infect Dis 2015;2:16-20.

40. Hartman TE, Primack SL, Müller NL, Staples CA.

Cite this article as: Guan C, Shi Y, Liu J, Yang Y, Zhang Q, Lu Z, Zheng G, Ye W, Xue M, Zhou X, Zhang N, Li H, Xie R, Chen B, Lu P. Pulmonary involvement in acquired immunodeficiency syndrome-associated Kaposi's sarcoma: a descriptive analysis of thin-section manifestations in 29 patients. Quant Imaging Med Surg 2021;11(2):714-724. doi: 10.21037/ qims-20-284
Diagnosis of thoracic complications in AIDS: accuracy of CT. AJR Am J Roentgenol 1994;162:547-53.

41. Del val Gómez MA, Castro Beiras JM, Gallardo FG, Verdejo J. Thallium and gallium scintigraphy in pulmonary Kaposi's sarcoma in an HIV-positive patient. Clin Nucl Med 1994;19:467-8. 